\title{
Secular trends in the serving sizes of published UK and Irish recipes
}

\author{
L. K. Pourshahidi, G. P. Faulkner, M. A. Kerr, T. A. McCrorie, J. M. W. Wallace \\ and M. B. E. Livingstone \\ Northern Ireland Centre for Food and Health, University of Ulster, Coleraine, BT52 1SA
}

The increasing portion sizes of foods available in the marketplace today have influenced the amount of food being served in the home ${ }^{(1,2)}$, and therefore overall energy intake ${ }^{(3)}$. Research from the US has recently demonstrated an increase in the serving size (SS) of classic recipes published in one cookbook over the last 70 years $^{(4)}$, however, it is unclear if similar trends are also apparent in the UK and Ireland. Therefore, the aim of the current study was to assess changes in the SS and energy content of recipes published in UK and Irish cookery books.

Forty-nine cookery books published between 1959 and 2010 were available, and common recipes were identified in the books published earlier (traditional) and those published more recently (recent). Ingredient weights in the recipes were standardised to grams ( $\mathrm{g}$ ) and recipes were entered into NetWISP (Tinuviel Software, Warrington, UK) for analysis of SS (g) and energy content (kJ per serving).

Common recipes were identified from 21 of the 49 cookery books and the mean difference in years between the traditional and recently published recipes was 27.5 years (range 11-46 years). Recipes were classified by type of meal (i.e. main meal, side dish or dessert).

\begin{tabular}{|c|c|c|c|c|c|c|c|}
\hline & \multirow[b]{3}{*}{$n$} & \multicolumn{4}{|c|}{ Recipes } & & \\
\hline & & \multicolumn{2}{|c|}{ Traditional } & \multicolumn{2}{|c|}{ Recent } & \multicolumn{2}{|c|}{ Difference* } \\
\hline & & Median & $\overline{\mathrm{IQR}}$ & Median & $\overline{\mathrm{IQR}}$ & Median & $\overline{\mathrm{IQR}}$ \\
\hline \multicolumn{8}{|l|}{ Serving Size $(\mathrm{g})$} \\
\hline All recipes & 23 & 313 & 264 & 281 & 176 & 21 & 150 \\
\hline Main meals & 13 & 367 & 83 & 404 & 241 & 47 & 90 \\
\hline Side Dishes & 6 & 133 & 295 & 152 & 118 & 3 & 143 \\
\hline Desserts & 4 & 157 & 62 & 233 & 46 & 51 & 54 \\
\hline
\end{tabular}

* Positive value means recipe has increased over time, and vice versa.

No significant differences between traditional and recent recipes $(P>0.05$, Mann-Whitney $\mathrm{U})$.

In all recipes included, there was a small non-significant increase in the median SS over time, which was most apparent within the main meals and dessert recipes. The median energy content per serving in the main meals and dessert recipes also showed a non-significant increase over time, by $12 \%$ and $55 \%$ respectively. Overall, $70 \%$ of the recipes increased in SS, and $61 \%$ increased in energy per serving over time.

In conclusion, a general pattern for increasing SS and consequently increasing energy content per serving was apparent from the small selection of recipes identified. Future studies should include additional recipes to confirm the observed increasing trends in commonly published recipes from the UK and Ireland.

This is based upon works supported by safefood, the Food Safety Promotion Board, under Grant No. 07-2010. We acknowledge Miss Helena Doherty, BSc Human Nutrition undergraduate student, for sourcing and analysing the recipes.

1. Young LR \& Nestle M (2003) J Am Diet Assoc 103, 231-4.

2. Wrieden W, Gregor A \& Barton K (2008) Proc Nut Soc 67, E211.

3. Duffey KJ \& Popkin BM (2011) PLoS Med 8, e1001050.

4. Wansink B \& Payne CR (2009) Ann Int Med 150, 291. 